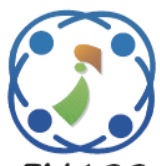

\title{
Integration of Group Method Analysis and Rough Sets Theory to Investigate Heat and Mass Transfer of the Flow of a Non-Newtonian Nanofluid towards a Vertical Stretching Surface
}

\author{
Hossam A. Nabwey ${ }^{1,2}$ \\ ${ }^{I}$ Department of Mathematics, College of Science and Humanities in Al-Kharj, \\ Prince Sattam bin Abdulaziz University, Al-Kharj11942, Saudi Arabia \\ ${ }^{2}$ Department of Basic Engineering Science, Faculty of Engineering, \\ Menoufia University, Shebin El-Kom,32511, Egypt \\ * Corresponding author's Email: eng_hossam21@yahoo.com
}

\begin{abstract}
This work reports how to utilize rough set theory and group method analysis for generating a set of rules to investigate heat and mass transfer of mixed convection stagnation point flow of a non-Newtonian nanofluid towards a vertical stretching surface. By utilizing group method analysis, the main partial differential equations which describe the flow are rehabilitated to nonlinear Ordinary Differential Equations (ODEs). Then, the resultant nonlinear ODEs are solved numerically by applying the implicit finite-difference scheme. The numerical values thus obtained are depicted in tabular form and the basic principles of rough sets are applied to get all reducts and finally a group of generalized rules are extracted to predict the value of local Nusselt number and local skin-friction coefficient. The outcomes demonstrate the novelty of the current work which can be summarized as hybridization of group method analysis and rough set theory to use in the field of fluid dynamics effectively. The resultant set of generalized classification rules which performed with basic logic functions can be considered as knowledge base with high accuracy and may be valuable in many engineering applications like power production, thermal extrusion systems and microelectronics.
\end{abstract}

Keywords: Classification, Rules extraction, Group method analysis, Nanofluid, Thermophoresis, Local musselt number, Local skin-friction coefficient, Rough sets theory, Feature selection.

\section{Introduction}

The research of non-Newtonian fluids is curiosity to numerous investigators because non-Newtonian fluids exhibit a nonlinear linkage among the rate of strain and stresses. This linkage leads to sophisticated rheological attitude which is not experienced at treating ordinary low-molecular-weight Newtonian fluids, since its behavior cannot be qualified by a single constitutive relation. The flow of nonNewtonian fluids also gains more interest due to its emerging in different applications in natural sciences, engineering sciences and industry. Many studies on these fluids are investigated by many researchers [1$6]$.
Nanofluids became a theme with considerable interest during the final decade due to their ability to improve the heat transfer rate in engineering suits. Nanofluids have wide utilities in cars and manufacturing cooling, tumefaction therapy, cooling towers, age of modern type fuels, cool down of microelectronics, cleverness of cross breed fueled engines and warming/cooling of home instruments. The pioneer experimental results of the researchers Choi and Eastman [7] was the first to utilize the word nanoparticle by keeping in mind the end goal to expand the heat conductivity of base liquids, how the warm conductivity is expanded is the main perspective of their work. The role of Brownian motion, interfacial resistance, morphology of suspended nanoparticles and aggregating behavior is 
investigated both experimentally and theoretically [8, 9], Then various exploratory and numerical examinations have been exhibited to explain the thermal and physical properties on different fluid flow patterns and heat transfer problems. Buongiorno [10] considered seven slip mechanisms that can produce a relative velocity between the nanoparticles and the base fluid. These are inertia, Brownian diffusion, thermophoresis, diffusiophoresis, Magnus effect, fluid drainage, and gravity. The 3D MHD nonlinear radiative hybrid nanofluid flow across an irregular dimension sheet with slip effect is studied numerically by Iskander [11]. Tiwari [12] investigated numerically the behavior of nanofluids is inside a two-sided lid-driven differentially heated square cavity to gain insight into convective recirculation and flow processes induced by a nanofluid. Ferdows [13] investigates the magnetohydrodynamic flow of a dissipative nanofluid, including gyrotactic microorganisms along an exponentially moving sheet. For more flow patterns see [14-29].

Group method and their invariants offer a powerful, sophisticated, and methodical technique to obtain the group-invariant solutions called selfsimilarity transformation. Self-similarity transformations make a reduct of the independent variables numbers of a set of partial Differential equations (PDEs), leading to convert the non-linear governing PDEs into the ODEs. Analysis using Lie group has been executed by many scientists and applied by mathematicians in many investigations. The linear transformation group approach is developed by Bakier [30] to simulate problem of hydromagnetic heat transfer by mixed convection along vertical plate in a liquid saturated porous medium in the presence of melting and thermal radiation effects for opposing external flow. Nabwey [31] used Lie Group Analysis to identify the characteristics of unsteady magnetohydrodynamic (MHD) flow of ferrofluid past a radiated stretching surface. More studies can be found in [32-36].

Rough sets theory (RST) is one of the successful approximations based mathematical model to deal the imprecision and uncertainty present in knowledge [37]. Many heuristic algorithms are proposed based on rough set theory, also numerous approaches based on rough set theory and other theories are investigated to extract decision rules and reduce the dimensionality of dataset. Nabwey [38] proposes a hybrid approach based on rough set methodology and fuzzy inference system for extraction of classification rules. Also in [39] he introduced Rough set theory based method for building knowledge for the rate of heat transfer on free convection over a vertical flat plate embedded in a porous medium. An approach based on Rough Sets Theory and Grey System for Implementation of Rule-Based Control for Sustainability of Rotary Clinker Kiln was introduced in [40]. Shaaban et al. [41, 42] used rough set methodology for steam turbine-generator fault diagnosis and for water reservoirs site location decision making, respectively. For more applications see [43-54]. One advantage of the rough set is the creation of readable if-then rules. Such rules have a potential to reveal new patterns in the data material. The main objective of this article is to inspect the effects of the Brownian motion and thermophoresis on mixed convection flow near stagnation point flow of a non-Newtonian nanofluid towards a vertical stretching surface, the model proposed by Buongiorno is employed for the nanofluid behavior. The basic governing partial Differential equations (PDEs) describing the problem are reduced into a similarity form using the one-parameter transformation group, and then the reduced similarity equations are solved numerically. Finally, the rough sets theory is applied to generate some classification rules to predict the different behaviors of relevant parameters on the local skin-friction coefficient and local Nusselt number. The proposed method put this work in a different position where we can obtain the values of local Nusselt number and local skin-friction coefficient at any values of the parameters under consideration and determine the most important parameters that affect the values of local Nusselt number and local skin-friction coefficient.

This paper is organized as follows: The Mathematical Framework of the problem is investigated in part two. Part three introduce the proposed methodology which is divided mainly two stages to extract the generalized decision rules. Then, the Outcomes and discussion are introduced and finally, concluded remarks are investigated.

\section{The mathematical framework}

According to [5] the mathematical model which describe the governing equations of steady, laminar, mixed convection, boundary layer flow of a nonNewtonian power-law nanofluid obeying the Ostwald-de Waele model [55] near stagnation-point at heated stretching vertical surface coinciding with the plane $y=0$ with the following assumptions : The flow being confined to the region $y=0$.

The model proposed by Buongiorno is employed for the nanofluid behavior in which the effects of Brownian motion and thermophoresis are taken into consideration. 
$U_{w}(x)=a x$, where $a$ is a constant

$U(x)=c x$ at $x=y=0$, where $c$ is a constant

and under usual assumptions in the literature, it can be written as:

$$
\begin{gathered}
\frac{\partial u}{\partial x}+\frac{\partial v}{\partial y}=0 \\
\rho_{f \infty}\left(u \frac{\partial u}{\partial x}+v \frac{\partial u}{\partial y}\right)=\rho_{f \infty} U \frac{d U}{d x}+\frac{\partial \tau_{x y}}{\partial y}+(1- \\
\left.C_{\infty}\right) \rho_{f \infty} g \beta\left(T-T_{\infty}\right)-\left(\rho_{p}-\rho_{f \infty}\right) g\left(C-C_{\infty}\right) \\
u \frac{\partial T}{\partial x}+v \frac{\partial T}{\partial y}=\alpha \frac{\partial^{2} T}{\partial y^{2}}+\tau\left[D_{B} \frac{\partial C}{\partial y} \frac{\partial T}{\partial y}+\frac{D_{T}}{T_{\infty}}\left(\frac{\partial T}{\partial y}\right)^{2}\right] \\
u \frac{\partial C}{\partial x}+v \frac{\partial C}{\partial y}=D_{B} \frac{\partial^{2} C}{\partial y^{2}}+\frac{D_{T}}{T_{\infty}} \frac{\partial^{2} T}{\partial y^{2}}
\end{gathered}
$$

Subject to the following boundary conditions:

$$
\begin{array}{ll}
\text { at } y=0 \quad & u=U_{w}(x), \quad v=0, \quad T=T_{w}, \\
& D_{B} \frac{\partial C}{\partial y}+\frac{D_{B}}{T_{\infty}} \frac{\partial T}{\partial y}=0
\end{array}
$$

as $\quad y \rightarrow \infty \quad u=U(x), \quad T=T_{\infty}, \quad C=C_{\infty}(6)$

With the aid of the following non-dimensional parameters:

$$
\begin{aligned}
& u=\frac{\partial \psi}{\partial y}, \quad v=-\frac{\partial \psi}{\partial x}, \quad \theta=\frac{T-T_{\infty}}{T_{w}-T_{\infty}} \\
& \varphi=\frac{C-C_{\infty}}{C_{\infty}}, \quad N r=\frac{\left(\rho_{p}-\rho_{f \infty}\right) C_{\infty}}{\rho_{f \infty} \beta\left(T_{w}-T_{\infty}\right)\left(1-C_{\infty}\right)}
\end{aligned}
$$

The mathematical model represented by Eqs. (1) - (4) can be reduced to:

$$
\begin{gathered}
\frac{\partial \psi}{\partial y} \frac{\partial^{2} \psi}{\partial x \partial y}-\frac{\partial \psi}{\partial x} \frac{\partial^{2} \psi}{\partial y^{2}}=a^{2} x+\frac{K}{\rho_{f \infty}} \frac{\partial}{\partial y}\left(\frac{\partial^{2} \psi}{\partial y^{2}}\right)^{n}+ \\
\left(1-C_{\infty}\right) \rho_{f \infty} g \beta\left(T_{w}-T_{\infty}\right)(\theta-N r \phi) \\
\frac{\partial \psi}{\partial y} \frac{\partial \theta}{\partial x}-\frac{\partial \psi}{\partial x} \frac{\partial \theta}{\partial y}=\alpha \frac{\partial^{2} \theta}{\partial y^{2}}+ \\
\tau\left[D_{B} C_{\infty} \frac{\partial \phi}{\partial y} \frac{\partial \theta}{\partial y}+\frac{D_{T}\left(T_{w}-T_{\infty}\right)}{T_{\infty}}\left(\frac{\partial \theta}{\partial y}\right)^{2}\right] \\
\frac{\partial \psi}{\partial y} \frac{\partial \phi}{\partial x}-\frac{\partial \psi}{\partial x} \frac{\partial \phi}{\partial y}=D_{B} \frac{\partial^{2} \phi}{\partial y^{2}}+\frac{D_{T}\left(T_{w}-T_{\infty}\right)}{T_{\infty} C_{\infty}} \frac{\partial^{2} \theta}{\partial y^{2}}(10)
\end{gathered}
$$

Subject to the following boundary conditions:

$$
\text { at } y=0 \quad \frac{\partial \psi}{\partial y}=c x, \frac{\partial \psi}{\partial x}=0, \quad \theta=1
$$

$$
\begin{aligned}
& D_{B} C_{\infty} \frac{\partial \varphi}{\partial y}+\frac{D_{B}\left(T_{w}-T_{\infty}\right)}{T_{\infty}} \frac{\partial \theta}{\partial y}=0 \\
& y \rightarrow \infty \quad \frac{\partial \psi}{\partial y}=a x, \quad \theta=0, \quad \varphi=0
\end{aligned}
$$

\section{Proposed methodology}

The proposed methodology consists of two main stages; the first stage is to use the one-parameter group to transform the mathematical model shown in (8) - (12) from PDE with two- independent variable to ODE in only one-independent variable While the second stage is to use the rough sets scheme to analyze, mining and generating generalized rules to predict the value of local Nusselt number, local skinfriction coefficient.

\subsection{The first stage}

This stage is initiated with the group G, a class of one-parameters ' $b$ ' of the form

$$
\begin{aligned}
& \bar{x}=C^{x}(b) x+K^{x}(b), \\
& \bar{y}=C^{y}(b) y+K^{y}(b), \\
& \bar{\psi}=C^{\psi}(b) \psi+K^{\psi}(b), \\
& \bar{\theta}=C^{\theta}(b) \theta+K^{\theta}(b), \\
& \bar{\varphi}=C^{\varphi}(b) \varphi+K^{\varphi}(b),
\end{aligned}
$$

where $\mathrm{Cs}$ and $\mathrm{Ks}$ are real valued and at least differentiable in their real argument $b$ and the transformations of the derivatives are obtained from $G$ via chain-rule operations

$$
\left.\begin{array}{c}
\bar{S}_{\bar{\imath}}=\left(C^{S} / C^{i}\right) S_{i} \\
\bar{S}_{\bar{\imath} \bar{j}}=\left(C^{S} / C^{i} C^{j}\right) S_{i j} \\
S_{\bar{\imath} \bar{j} \bar{k}}=\left(C^{S} / C^{i} C^{j} C^{k}\right) S_{i j k}
\end{array}\right\} i, j, k=x, y
$$

where $S$ stands for $\psi, \theta, \phi$. Which summarize in a group $G$ to transform invariantly the governing Eqs. (8) - (12) (for more Details see [5]) of the form:

$$
G=\left\{\begin{array}{c}
G_{s}=\left\{\begin{array}{c}
\bar{x}=\left(C^{y}\right)^{2} x, \\
\bar{y}=C^{y} y,
\end{array}\right. \\
\left\{\begin{array}{c}
\bar{\psi}=C^{y} \psi+K^{\psi}, \\
\bar{\theta}=\theta, \\
\bar{\varphi}=\varphi .
\end{array}\right.
\end{array}\right.
$$

The next step is to apply the basic theorem in group theory (see Moran and Gaggioli [56]) to find the complete set of absolute invariants which are the absolute invariants of the independent variables $(x, y)$ is $\eta=\eta(x, y)$, and the absolute invariants of the dependent variables $(\psi, \theta, \phi)$ are $\psi(x, y)=$ 
$\xi(x) F(\eta), \theta(x, y)=\theta(\eta), \phi(x, y)=\phi(\eta)$. Finally, reduce the governing equations to a set of ordinary differential equations in $F(\eta), \theta(\eta)$ and $\phi(\eta)$ and hence transfer it to a set of ordinary differential equations in a single variable $\eta$ by making the coefficients of the functions $F(\eta), \theta(\eta), \phi(\eta)$ and their derivatives, be an arbitrary constants or functions of $\eta$ only. Yields the mathematical model:

$$
\begin{aligned}
& n\left(F^{\prime \prime}\right)^{n-1} F^{\prime \prime \prime}+\left(\frac{2 n}{n+1}\right) F F^{\prime \prime}-F^{2}+\frac{a^{2}}{c^{2}}+ \\
& \Lambda(\theta-N r \phi)=0 \\
& \theta^{\prime \prime}+\operatorname{Pr}\left(\frac{2 n}{n+1} F \theta^{\prime}+N b \theta^{\prime} \phi^{\prime}+N t \theta^{\prime 2}\right)=0 \\
& \phi^{\prime \prime}+L e \frac{2 n}{n+1} F \phi^{\prime}+\frac{N t}{N b} \theta^{\prime \prime}=0
\end{aligned}
$$

Subject to the following boundary conditions:

$$
\begin{gathered}
F(0)=0 \quad F^{\prime}(0)=1 \quad \theta(0)=1 \\
N b \varphi^{\prime}(0)+N t \theta^{\prime}(0)=0 \\
F^{\prime}(\infty)=\frac{a}{c}, \theta(\infty)=\phi(\infty)=0
\end{gathered}
$$

Where

$$
\lambda=\frac{G r_{x}}{R e_{x}^{2}} \operatorname{Pr}=\frac{c x^{2}}{\alpha} R e_{x}^{-2 /(n+1)} \operatorname{Re} e_{x}=\frac{(c x)^{2-n} x^{n}}{K / \rho_{f \infty}}
$$

$$
\begin{array}{r}
N b=\frac{\tau D_{B} C_{\infty}}{c x^{2} R e_{x}^{-\frac{2}{n+1}}} N t=\frac{\tau D_{T}\left(T_{w}-T_{\infty}\right)}{T_{\infty} c x^{2} R e_{x}^{-\frac{2}{n+1}}} \\
L e=\frac{c x^{2}}{D_{B}} R e_{x}^{-2 /(n+1)} \quad G r_{x}=\frac{\left(1-C_{\infty}\right) \rho_{f \infty} g \beta\left(T_{w}-T_{\infty}\right)}{\left(K / \rho_{f \infty}\right)^{2}}
\end{array}
$$

It is worth noting that the differentiation in (16) (20) with respect to $\eta$ and all the details can be found in [5].

The most significant parameters for this paper are the local skin friction $C_{f}$ and local Nusselt number $\mathrm{Nu}_{\mathrm{x}}$, and these quantities can be written as:

$$
\begin{aligned}
& C_{f}=2\left(F^{\prime \prime}(0)\right)^{n}\left[\frac{(c x)^{2-n} x^{n}}{K / \rho_{f \infty}}\right]^{-1 /(1+n)} \\
& N u_{x}=-\left(\frac{u_{w}^{2-n}}{K / \rho_{f \infty}}\right)^{1 /(1+n)} \theta^{\prime}(0)
\end{aligned}
$$

Where the local skin friction factor $\mathrm{Cf}$ is proportional to the numerical values of $\left(\mathrm{F}^{\prime \prime}(0)\right) \mathrm{n}$ and and the local Nusselt number Nux is proportional to the numerical values of $\theta^{\prime}(0)$.

Now it is required to obtain the solution of the Eqs. (16) - (18) subject to the boundary conditions in Eqs. (19) and (20). since formulated equations are highly nonlinear and coupled their analytical solutions are difficult, it is solved numerically with the aid of the

Table 1. Decision table of local skin friction coefficient for various values of $\mathrm{Nr}, \mathrm{Nb}, \mathrm{Nt}, \mathrm{n}, \Lambda, \mathrm{a} / \mathrm{c}$ at $\operatorname{Pr}=6.8$ and $\mathrm{Le}=10$

\begin{tabular}{rrrrrrrr}
\hline $\mathrm{U}$ & $\mathrm{Nr}$ & $\mathrm{Nb}$ & $\mathrm{Nt}$ & $\mathrm{n}$ & $\Lambda$ & $a / c$ & $\begin{array}{r}\text { local skin friction coefficient } \\
\left(\mathrm{F}^{\prime \prime}(0)\right)^{\mathrm{n}}\end{array}$ \\
\hline $\mathrm{X} 1$ & 0.1 & 0.5 & 0.3 & 0.5 & 3.0 & 1.5 & 1.797508 \\
$\mathrm{X} 2$ & 0.3 & 0.5 & 0.3 & 0.5 & 3.0 & 1.5 & 1.792772 \\
$\mathrm{X} 3$ & 0.5 & 0.5 & 0.3 & 0.5 & 3.0 & 1.5 & 1.788013 \\
$\mathrm{X} 4$ & 0.1 & 0.1 & 0.3 & 0.5 & 3.0 & 1.5 & 1.788109 \\
$\mathrm{X} 5$ & 0.1 & 0.3 & 0.3 & 0.5 & 3.0 & 1.5 & 1.795914 \\
$\mathrm{X} 6$ & 0.1 & 0.5 & 0.3 & 0.5 & 3.0 & 1.5 & 1.797508 \\
$\mathrm{X} 7$ & 0.1 & 0.5 & 0.1 & 0.5 & 3.0 & 1.5 & 1.727497 \\
$\mathrm{X} 8$ & 0.1 & 0.5 & 0.3 & 0.5 & 3.0 & 1.5 & 1.797508 \\
$\mathrm{X} 9$ & 0.1 & 0.5 & 0.5 & 0.5 & 3.0 & 1.5 & 1.849623 \\
$\mathrm{X} 10$ & 0.1 & 0.5 & 0.3 & 0.5 & 3.0 & 1.5 & 1.797508 \\
$\mathrm{X} 11$ & 0.1 & 0.5 & 0.3 & 1.0 & 3.0 & 1.5 & 1.700608 \\
$\mathrm{X} 12$ & 0.1 & 0.5 & 0.3 & 1.5 & 3.0 & 1.5 & 0.399424 \\
$\mathrm{X} 13$ & 0.1 & 0.5 & 0.3 & 0.5 & -2.0 & 1.5 & 1.352609 \\
$\mathrm{X} 14$ & 0.1 & 0.5 & 0.3 & 0.5 & 1.0 & 1.5 & 1.587690 \\
$\mathrm{X} 15$ & 0.1 & 0.5 & 0.3 & 0.5 & 2.0 & 1.5 & 2.797508 \\
$\mathrm{X} 16$ & 0.1 & 0.5 & 0.3 & 0.5 & 3.0 & 1.5 & 2.301840 \\
$\mathrm{X} 17$ & 0.1 & 0.5 & 0.3 & 0.5 & 3.0 & 1.7 & \\
$\mathrm{X} 18$ & 0.1 & 0.5 & 0.3 & 0.5 & 3.0 & 1.9 & \\
\hline
\end{tabular}


Received: August 14, 2020. Revised: September 3, 2020.

Table 2. Decision table of local nusselt number for various values of $\mathrm{Nr}, \mathrm{Nb}, \mathrm{Nt}, \mathrm{n}, \Lambda, \mathrm{a} / \mathrm{c}$ at $\operatorname{Pr}=6.8$ and $\mathrm{Le}=10$

\begin{tabular}{|c|c|c|c|c|c|c|c|}
\hline $\mathrm{U}$ & $\mathrm{Nr}$ & $\mathrm{Nb}$ & $\mathrm{Nt}$ & $\mathrm{n}$ & $\Lambda$ & $a / c$ & $\begin{array}{r}\text { local Nusselt number } \\
-\theta^{\prime}(0)\end{array}$ \\
\hline $\mathrm{X} 1$ & 0.1 & 0.5 & 0.3 & 0.5 & 3.0 & 1.5 & 0.825806 \\
\hline $\mathrm{X} 2$ & 0.3 & 0.5 & 0.3 & 0.5 & 3.0 & 1.5 & 0.824981 \\
\hline $\mathrm{X} 3$ & 0.5 & 0.5 & 0.3 & 0.5 & 3.0 & 1.5 & 0.824468 \\
\hline $\mathrm{X} 4$ & 0.1 & 0.1 & 0.3 & 0.5 & 3.0 & 1.5 & 0.824515 \\
\hline $\mathrm{X} 5$ & 0.1 & 0.3 & 0.3 & 0.5 & 3.0 & 1.5 & 0.825869 \\
\hline X6 & 0.1 & 0.5 & 0.3 & 0.5 & 3.0 & 1.5 & 0.825806 \\
\hline $\mathrm{X} 7$ & 0.1 & 0.5 & 0.1 & 0.5 & 3.0 & 1.5 & 1.391290 \\
\hline $\mathrm{X} 8$ & 0.1 & 0.5 & 0.3 & 0.5 & 3.0 & 1.5 & 0.825806 \\
\hline X9 & 0.1 & 0.5 & 0.5 & 0.5 & 3.0 & 1.5 & 0.554399 \\
\hline $\mathrm{X} 10$ & 0.1 & 0.5 & 0.3 & 0.5 & 3.0 & 1.5 & 0.825806 \\
\hline $\mathrm{X} 11$ & 0.1 & 0.5 & 0.3 & 1.0 & 3.0 & 1.5 & 0.975382 \\
\hline $\mathrm{X} 12$ & 0.1 & 0.5 & 0.3 & 1.5 & 3.0 & 1.5 & 1.056164 \\
\hline X13 & 0.1 & 0.5 & 0.3 & 0.5 & -2.0 & 1.5 & 0.752429 \\
\hline X14 & 0.1 & 0.5 & 0.3 & 0.5 & 1.0 & 1.5 & 0.797467 \\
\hline $\mathrm{X} 15$ & 0.1 & 0.5 & 0.3 & 0.5 & 2.0 & 1.5 & 0.812213 \\
\hline $\mathrm{X} 16$ & 0.1 & 0.5 & 0.3 & 0.5 & 3.0 & 1.5 & 0.825806 \\
\hline X17 & 0.1 & 0.5 & 0.3 & 0.5 & 3.0 & 1.7 & 0.854231 \\
\hline $\mathrm{X} 18$ & 0.1 & 0.5 & 0.3 & 0.5 & 3.0 & 1.9 & 0.88326 \\
\hline
\end{tabular}

implicit, tri-diagonal, finite-difference scheme. The numerical results are reported in Table 1 and Table 2 which represent the decision tables for local skin friction factor $\left(F^{\prime \prime}(0)\right) n$, local Nusselt number $-\theta^{\prime}(0)$, respectively [5].

\subsection{The second stage}

Rough sets schem in this stage, we will apply the basic principles of Rough sets theory to discover structural relationships within the given training data by establishment of equivalence classes then a definition for a given class is approximated by two sets, namely, a lower approximation of and an upper approximation as shown in Fig. 1 where each rectangular region represents an equivalence class. Finally, computation of reducts from data and derivation of rules are done.

The proposed rough sets methodology will be used to mining and generating generalized rules to predict the value of local skin friction coefficient and

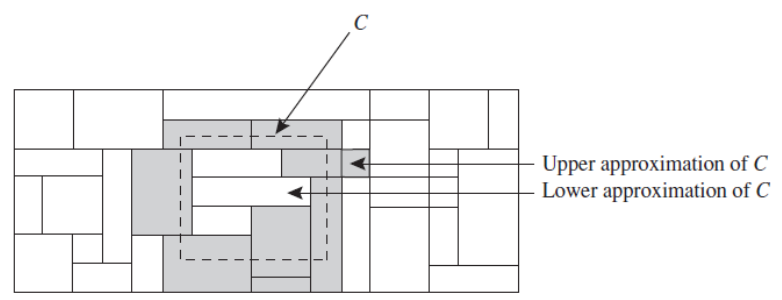

Figure. 1 Graphical representation of lower and upper approximation

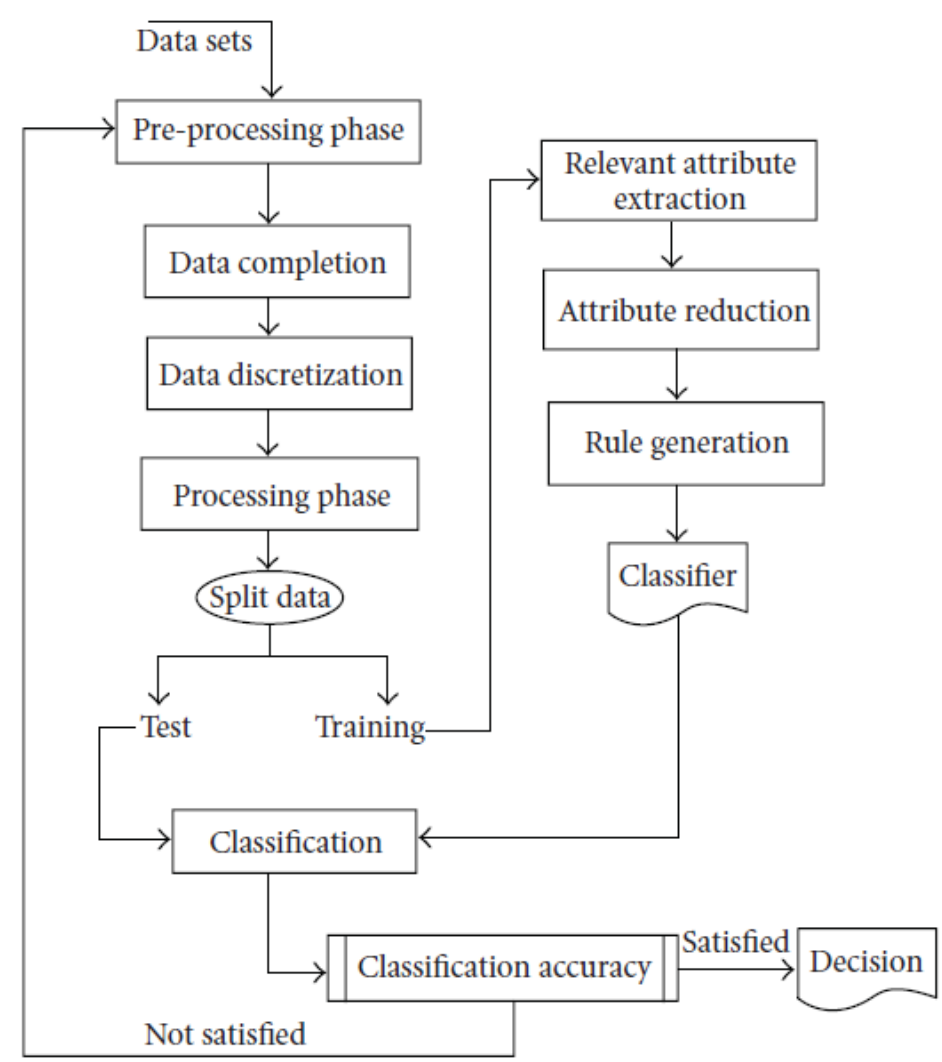

Figure. 2 The overall steps of the second stage

local Nusselt number for various values of $\mathrm{Nr}, \mathrm{Nb}, \mathrm{Nt}$, $\mathrm{n}, \Lambda, \mathrm{a} / \mathrm{c}$. the overall steps of this stage are shown in Fig. 2. 


\section{Outcomes and discussion}

By using software called ROSETTA which is an RST analysis toolkit, rough sets with Boolean reasoning discretization algorithm is introduced to discretize the data as shown in Table 3 and Table 4 where * means do not care condition. Next step is to find the equivalence classes as shown in Table 5, later the rough set reduction technique is applied to find the minimal subsets (reducts) of attributes that can describe all the concepts in the decision tables as shown in Table 6. Finally, the knowledge gained from all generated reducts can be represented by rough sets dependency rules as shown in Table 7 and Table 8.

Table 3. The discretized decision table of Table 1.

\begin{tabular}{|c|c|c|c|c|c|c|c|}
\hline $\mathrm{U}$ & $\mathrm{Nr}$ & $\mathrm{Nb}$ & $\mathrm{Nt}$ & $\mathrm{n}$ & $\Lambda$ & $a / c$ & $\begin{array}{l}\text { local skin friction } \\
\text { coefficient }\left(F^{\prime \prime}(0)\right)^{\mathrm{n}}\end{array}$ \\
\hline $\mathrm{X} 1$ & $\left(0.2,{ }^{*}\right]$ & $(*, 0.4]$ & $(0.4,0.2]$ & $(0.8, *]$ & $(*, 3]$ & $\left(1.6,{ }^{*}\right]$ & 1.79751 \\
\hline $\mathrm{X} 2$ & $(0.4,0.2]$ & $(*, 0.4]$ & $(0.4,0.2]$ & $(0.8, *]$ & $(*, 3]$ & $\left(1.6,{ }^{*}\right]$ & 1.79277 \\
\hline X3 & $(*, 0.4]$ & $(*, 0.4]$ & $(0.4,0.2]$ & $(0.8, *]$ & $(*, 3]$ & $\left(1.6,{ }^{*}\right]$ & 1.78801 \\
\hline $\mathrm{X} 4$ & $(0.2, *]$ & $(0.2, *]$ & $(0.4,0.2]$ & $(0.8, *]$ & $(*, 3]$ & $\left(1.6,{ }^{*}\right]$ & 1.78811 \\
\hline X5 & $(0.2, *]$ & $(0.4,0.2]$ & $(0.4,0.2]$ & $(0.8, *]$ & $(*, 3]$ & $\left(1.6,{ }^{*}\right]$ & 1.79591 \\
\hline X6 & $(0.2, *]$ & $(*, 0.4]$ & $(0.4,0.2]$ & $(0.8, *]$ & $(*, 3]$ & $\left(1.6,{ }^{*}\right]$ & 1.79751 \\
\hline X7 & $(0.2, *]$ & $(*, 0.4]$ & $(0.2, *]$ & $(0.8, *]$ & $(*, 3]$ & $\left(1.6,{ }^{*}\right]$ & 1.72750 \\
\hline X8 & $(0.2, *]$ & $(*, 0.4]$ & $(0.4,0.2]$ & $(0.8, *]$ & $(*, 3]$ & $\left(1.6,{ }^{*}\right]$ & 1.79751 \\
\hline X9 & $(0.2, *]$ & $(*, 0.4]$ & $(*, 0.4]$ & $(0.8, *]$ & $(*, 3]$ & $\left(1.6,{ }^{*}\right]$ & 1.84962 \\
\hline X10 & $(0.2, *]$ & $(*, 0.4]$ & $(0.4,0.2]$ & $(0.8, *]$ & $(*, 3]$ & $\left(1.6,{ }^{*}\right]$ & 1.79751 \\
\hline X11 & $(0.2, *]$ & $(*, 0.4]$ & $(0.4,0.2]$ & $(1.3,0.8]$ & $(*, 3]$ & $\left(1.6,{ }^{*}\right]$ & 1.70061 \\
\hline X12 & $(0.2, *]$ & $(*, 0.4]$ & $(0.4,0.2]$ & $(*, 1.3]$ & $(*, 3]$ & $\left(1.6,{ }^{*}\right]$ & 1.64715 \\
\hline X13 & $(0.2, *]$ & $(*, 0.4]$ & $(0.4,0.2]$ & $(0.8, *]$ & $(0, *]$ & $\left(1.6,{ }^{*}\right]$ & 0.39942 \\
\hline X14 & $(0.2, *]$ & $(*, 0.4]$ & $(0.4,0.2]$ & $(0.8, *]$ & $(2,0]$ & $\left(1.6,{ }^{*}\right]$ & 1.35261 \\
\hline X15 & $(0.2, *]$ & $(*, 0.4]$ & $(0.4,0.2]$ & $(0.8, *]$ & $(3,2]$ & $\left(1.6,{ }^{*}\right]$ & 1.58769 \\
\hline X16 & $(0.2, *]$ & $(*, 0.4]$ & $(0.4,0.2]$ & $(0.8, *]$ & $(*, 3]$ & $\left(1.6,{ }^{*}\right]$ & 1.79751 \\
\hline X17 & $(0.2, *]$ & $(*, 0.4]$ & $(0.4,0.2]$ & $(0.8, *]$ & $(*, 3]$ & $(1.8,1.6]$ & 2.05290 \\
\hline $\mathrm{X} 18$ & $(0.2, *]$ & $(*, 0.4]$ & $(0.4,0.2]$ & $(0.8, *]$ & $(*, 3]$ & $(*, 1.8]$ & 2.30184 \\
\hline
\end{tabular}

Table 4. The discretized decision table of Table 2

\begin{tabular}{|c|c|c|c|c|c|c|c|}
\hline $\mathrm{U}$ & $\mathrm{Nr}$ & $\mathrm{Nb}$ & $\mathrm{Nt}$ & $\mathrm{n}$ & $\Lambda$ & $a / c$ & $\begin{array}{r}\text { local Nusselt number } \\
-\theta^{\prime}(0)\end{array}$ \\
\hline $\mathrm{X} 1$ & $\left(0.2,{ }^{*}\right]$ & $(*, 0.4]$ & $(0.4,0.2]$ & $\left(0.8,{ }^{*}\right]$ & $(*, 3]$ & $\left(1.6,{ }^{*}\right]$ & 1.79751 \\
\hline $\mathrm{X} 2$ & $(0.4,0.2]$ & $(*, 0.4]$ & $(0.4,0.2]$ & $(0.8, *]$ & $(*, 3]$ & $\left(1.6,{ }^{*}\right]$ & 1.79277 \\
\hline X3 & $(*, 0.4]$ & $(*, 0.4]$ & $(0.4,0.2]$ & $(0.8, *]$ & $(*, 3]$ & $\left(1.6,{ }^{*}\right]$ & 1.78801 \\
\hline $\mathrm{X} 4$ & $(0.2, *]$ & $(0.2, *]$ & $(0.4,0.2]$ & $(0.8, *]$ & $(*, 3]$ & $\left(1.6,{ }^{*}\right]$ & 1.78811 \\
\hline X5 & $(0.2, *]$ & $(0.4,0.2]$ & $(0.4,0.2]$ & $(0.8, *]$ & $(*, 3]$ & $\left(1.6,{ }^{*}\right]$ & 1.79591 \\
\hline X6 & $(0.2, *]$ & $(*, 0.4]$ & $(0.4,0.2]$ & $(0.8, *]$ & $(*, 3]$ & $\left(1.6,{ }^{*}\right]$ & 1.79751 \\
\hline $\mathrm{X} 7$ & $(0.2, *]$ & $(*, 0.4]$ & $(0.2, *]$ & $(0.8, *]$ & $(*, 3]$ & $\left(1.6,{ }^{*}\right]$ & 1.72750 \\
\hline $\mathrm{X} 8$ & $(0.2, *]$ & $(*, 0.4]$ & $(0.4,0.2]$ & $(0.8, *]$ & $(*, 3]$ & $\left(1.6,{ }^{*}\right]$ & 1.79751 \\
\hline X9 & $(0.2, *]$ & $(*, 0.4]$ & $(*, 0.4]$ & $(0.8, *]$ & $(*, 3]$ & $\left(1.6,{ }^{*}\right]$ & 1.84962 \\
\hline X10 & $(0.2, *]$ & $(*, 0.4]$ & $(0.4,0.2]$ & $(0.8, *]$ & $(*, 3]$ & $(1.6, *]$ & 1.79751 \\
\hline $\mathrm{X} 11$ & $(0.2, *]$ & $(*, 0.4]$ & $(0.4,0.2]$ & $(1.3,0.8]$ & $(*, 3]$ & $\left(1.6,{ }^{*}\right]$ & 1.70061 \\
\hline $\mathrm{X} 12$ & $(0.2, *]$ & $(*, 0.4]$ & $(0.4,0.2]$ & $(*, 1.3]$ & $(*, 3]$ & $(1.6, *]$ & 1.64715 \\
\hline $\mathrm{X} 13$ & $(0.2, *]$ & $(*, 0.4]$ & $(0.4,0.2]$ & $(0.8, *]$ & $(0, *]$ & $\left(1.6,{ }^{*}\right]$ & 0.39942 \\
\hline X14 & $\left(0.2,{ }^{*}\right]$ & $(*, 0.4]$ & $(0.4,0.2]$ & $(0.8, *]$ & $(2,0]$ & $\left(1.6,{ }^{*}\right]$ & 1.35261 \\
\hline X15 & $(0.2, *]$ & $(*, 0.4]$ & $(0.4,0.2]$ & $(0.8, *]$ & $(3,2]$ & $\left(1.6,{ }^{*}\right]$ & 1.58769 \\
\hline X16 & $(0.2, *]$ & $(*, 0.4]$ & $(0.4,0.2]$ & $(0.8, *]$ & $(*, 3]$ & $\left(1.6,{ }^{*}\right]$ & 1.79751 \\
\hline X17 & $(0.2, *]$ & $(*, 0.4]$ & $(0.4,0.2]$ & $(0.8, *]$ & $(*, 3]$ & $(1.8,1.6]$ & 2.05290 \\
\hline $\mathrm{X} 18$ & $(0.2, *]$ & $(*, 0.4]$ & $(0.4,0.2]$ & $(0.8, *]$ & $(*, 3]$ & $(*, 1.8]$ & 2.30184 \\
\hline
\end{tabular}


Table 5. The equivalence classes of discretized decision table

\begin{tabular}{lllllllll}
\hline Eq. Classes & $\{4\}$ & $\{5\}$ & $\{7\}$ & $\{13\}$ & $\{14\}$ & $\{15\}$ & $\{1,6,8,10,16\}$ & $\{17\}$ \\
\hline Cardinality & 1 & 1 & 1 & 1 & 1 & 1 & 5 & 5 \\
& & & & & & & & \\
Eq. Classes & $\{18\}$ & $\{11\}$ & $\{12\}$ & $\{9\}$ & $\{2\}$ & $\{3\}$ & $\{11\}$ \\
Cardinality & 1 & 1 & 1 & 1 & & & 1 & \\
\hline
\end{tabular}

Table 6. Reducts of discretized decision table

\begin{tabular}{llllllll}
\hline Reduct & $\{\mathrm{Nr}, \mathrm{Nb}, \mathrm{Nt}, \mathrm{n}, \Lambda, \mathrm{a} / \mathrm{c}\}$ & $\{\mathrm{Nr}\}$ & $\{\mathrm{Nb}\}$ & $\{\mathrm{Nt}\}$ & $\{\mathrm{n}\}$ & $\{\Lambda\}$ & $\{\mathrm{a} / \mathrm{c}\}$ \\
\hline Support & 100 & 100 & 100 & 100 & 100 & 100 & 100 \\
Length & 6 & 1 & 1 & 1 & 1 & 1 & 1 \\
\hline
\end{tabular}

Table 7. The generated rules to predict the value of local skin friction coefficient $\left(F^{\prime \prime}(0)\right)^{n}$

\begin{tabular}{|l|l|l|l|l|l|}
\hline & Rule & $\begin{array}{l}\text { LHS } \\
\text { SUPPORT }\end{array}$ & $\begin{array}{l}\text { RHS } \\
\text { SUPPORT }\end{array}$ & $\begin{array}{l}\text { RHS } \\
\text { ACCURACY }\end{array}$ & $\begin{array}{l}\text { LHS } \\
\text { COVERAGE }\end{array}$ \\
\hline & $\begin{array}{l}\text { If } \mathrm{Nr}([*, 0.2)) \text { AND Nb }([0.4, *)) \text { AND } \\
\mathrm{Nt}([0.2,0.4)) \text { AND }, \mathrm{n}\left(\left[^{*}, 0.8\right)\right) \text { AND } \\
\Lambda([3, *)) \text { AND a/c }([*, 1.6))=>\left(\mathrm{F}^{\prime \prime}(0)\right)^{\mathrm{n}} \\
(1.79751)\end{array}$ & 5 & 5 & 1.0 & 0.277778 \\
\hline 2 & If $\mathrm{Nr}([0.2,0.4))=>\left(\mathrm{F}^{\prime \prime}(0)\right)^{\mathrm{n}}(1.79277)$ & 1 & 1 & 1.0 & 0.055556 \\
\hline 3 & If $\mathrm{Nr}([0.4, *))=>\left(\mathrm{F}^{\prime \prime}(0)\right)^{\mathrm{n}}(1.78801)$ & 1 & 1 & 1.0 & 0.055556 \\
\hline 4 & If $\mathrm{Nb}([*, 0.2))=>\left(\mathrm{F}^{\prime \prime}(0)\right)^{\mathrm{n}}(1.78811)$ & 1 & 1 & 1.0 & 0.055556 \\
\hline 5 & If $\mathrm{Nb}([0.2,0.4))=>\left(\mathrm{F}^{\prime \prime}(0)\right)^{\mathrm{n}}(1.79591)$ & 1 & 1 & 1.0 & 0.055556 \\
\hline 6 & If $\mathrm{Nt}([*, 0.2))=>\left(\mathrm{F}^{\prime \prime}(0)\right)^{\mathrm{n}}(1.72750)$ & 1 & 1 & 1.0 & 0.055556 \\
\hline 7 & If $\mathrm{Nt}([0.4, *))=>\left(\mathrm{F}^{\prime \prime}(0)\right)^{\mathrm{n}}(1.84962)$ & 1 & 1 & 1.0 & 0.055556 \\
\hline 8 & If $\mathrm{n}([0.8,1.3))=>\left(\mathrm{F}^{\prime \prime}(0)\right)^{\mathrm{n}}(1.70061)$ & 1 & 1 & 1.0 & 0.055556 \\
\hline 9 & If $\mathrm{n}([1.3, *))=>\left(\mathrm{F}^{\prime \prime}(0)\right)^{\mathrm{n}}(1.64715)$ & 1 & 1 & 1.0 & 0.055556 \\
\hline 10 & If $\Lambda([*, 0))=>\left(\mathrm{F}^{\prime \prime}(0)\right)^{\mathrm{n}}(0.39942)$ & 1 & 1 & 1.0 & 0.055556 \\
\hline 11 & If $\Lambda([0,2))=>\left(\mathrm{F}^{\prime \prime}(0)\right)^{\mathrm{n}}(1.35261)$ & 1 & 1 & 1.0 & 0.055556 \\
\hline 12 & If $\Lambda([2,3))=>\left(\mathrm{F}^{\prime \prime}(0)\right)^{\mathrm{n}}(1.58769)$ & 1 & 1 & 1.0 & 0.055556 \\
\hline 13 & If a/c $([1.6,1.8))=>\left(\mathrm{F}^{\prime \prime}(0)\right)^{\mathrm{n}}(2.05290)$ & 1 & 1 & 1.0 & 0.055556 \\
\hline 14 & If a/c $([1.8, *))=>\left(\mathrm{F}^{\prime \prime}(0)\right)^{\mathrm{n}}(2.30184)$ & 1 & 1 & 1.0 & 0.055556 \\
\hline
\end{tabular}

As shown in Tables 7 and 8 , the extracted decision rules represent the impact of some thermophysical parameters such as thermophoresis parameter, power-law index parameter, ratio of velocity parameter, Brownian motion parameter, nanoparticle buoyancy ratio and mixed convection parameter on the local skin friction coefficient $\left(F^{\prime \prime}(0)\right)^{n}$ and local Nusselt number $-\theta^{\prime}(0)$. It is found that as the power-index increases there is an increase in - $\theta^{\prime}(0)$ and decrease in $\left(F^{\prime \prime}(0)\right)^{n}$ due to the reduction of the thermal boundary-layer thickness which leads to decreasing in the nanofluid velocity, this produce a depress in the wall shear stress and improvement of rate of heat transfer at the surface. as well as the increase in the velocity ratio and mixed convection parameters result in accelerating the flow which in turn cause a reduction in the fluid temperature and increases the maximum velocity leading to the increase of $\left(F^{\prime \prime}(0)\right)^{n}$ and $-\theta^{\prime}(0)$. It is also noted that a slight reduction in $\left(F^{\prime \prime}(0)\right)^{n}$ and $-\theta^{\prime}(0)$ is occurred due to the increase in nanoparticle buoyancy ratio. Moreover a slight enhancement in $\left(F^{\prime \prime}(0)\right)^{n}$ and $-\theta^{\prime}(0)$ is occurred as the Brownian parameter increased because Brownian diffusion increase heat conduction. In order to check the effectiveness of the proposed method and test the accuracy of the obtained results, the results for skin friction coefficient $F^{\prime \prime}(0)$ are compared with those of Wang [57], Gorla and Sidawi [58] and Nabwey et al. [5] for different values of the related parameters as shown in Table 9. From these comparisons it is clear that there is an excellent agreement and the accuracy of the proposed method is high.

This work introduced a methodology based on group method analysis and rough sets theory for generating a set of decision rules to investigate and predict the value of local Nusselt number and local skin-friction coefficient considering the effects of the Brownian motion and thermophoresis on mixed convection stagnation point flow of a Non-Newtonian 
Table 8.The generated rules to predict the value of local nusselt number $-\theta^{\prime}(0)$

\begin{tabular}{|l|l|l|l|l|l|}
\hline & Rule & $\begin{array}{l}\text { LHS } \\
\text { SUPPORT }\end{array}$ & $\begin{array}{l}\text { RHS } \\
\text { SUPPORT }\end{array}$ & $\begin{array}{l}\text { RHS } \\
\text { ACCURACY }\end{array}$ & $\begin{array}{l}\text { LHS } \\
\text { COVERAGE }\end{array}$ \\
\hline & $\begin{array}{l}\text { If } \mathrm{Nr}([*, 0.2)) \text { AND Nb }([0.4, *)) \text { AND } \\
\mathrm{Nt}([0.2,0.4)) \text { AND }, \mathrm{n}\left(\left[{ }^{*}, 0.8\right)\right) \text { AND } \\
\Lambda([3, *)) \text { AND a/c }([*, 1.6))=>-\theta^{\prime}(0) \\
(0.82581)\end{array}$ & 5 & 5 & 1.0 & 0.277778 \\
\hline 2 & If $\mathrm{Nr}([0.2,0.4))=>-\theta^{\prime}(0)(0.82498)$ & 1 & 1 & 1.0 & 0.055556 \\
\hline 3 & If $\mathrm{Nr}([0.4, *))=>-\theta^{\prime}(0)(0.82447)$ & 1 & 1 & 1.0 & 0.055556 \\
\hline 4 & If $\mathrm{Nb}([*, 0.2))=>-\theta^{\prime}(0)(0.82451)$ & 1 & 1 & 1.0 & 0.055556 \\
\hline 5 & If $\mathrm{Nb}([0.2,0.4))=>-\theta^{\prime}(0)(0.82587)$ & 1 & 1 & 1.0 & 0.055556 \\
\hline 6 & If $\mathrm{Nt}\left(\left[*^{*}, 0.2\right)\right)=>-\theta^{\prime}(0)(1.39129)$ & 1 & 1 & 1.0 & 0.055556 \\
\hline 7 & If $\mathrm{Nt}([0.4, *))=>-\theta^{\prime}(0)(0.55440)$ & 1 & 1 & 1.0 & 0.055556 \\
\hline 8 & If $\mathrm{n}([0.8,1.3))=>-\theta^{\prime}(0)(0.97538)$ & 1 & 1 & 1.0 & 0.055556 \\
\hline 9 & If $\mathrm{n}([1.3, *))=>-\theta^{\prime}(0)(1.05616)$ & 1 & 1 & 1.0 & 0.055556 \\
\hline 10 & If $\Lambda([*, 0))=>-\theta^{\prime}(0)(0.75243)$ & 1 & 1 & 1.0 & 0.055556 \\
\hline 11 & If $\Lambda([0,2))=>-\theta^{\prime}(0)(0.79747)$ & 1 & 1 & 1.0 & 0.055556 \\
\hline 12 & If $\Lambda([2,3))=>-\theta^{\prime}(0)(0.81221)$ & 1 & 1 & 1.0 & 0.055556 \\
\hline 13 & If $\mathrm{a} / \mathrm{c}([1.6,1.8))=>-\theta^{\prime}(0)(0.85423)$ & 1 & 1 & 1.0 & 0.055556 \\
\hline 14 & If a/c $([1.8, *))=>-\theta^{\prime}(0)(0.88326)$ & 1 & 1 & 1.0 & 0.055556 \\
\hline
\end{tabular}

Table 9. Comparison of $F^{\prime \prime}(0)$ for various values of $\operatorname{Pr}$ at $\mathrm{n}=1, \Lambda=\mathrm{Nr}=0$

\begin{tabular}{llllll}
\hline $\mathrm{U}$ & $\operatorname{Pr}$ & Wang [57] & Gorla and Sidawi [58] & Nabwey et al. [5] & Current work \\
\hline $\mathrm{X} 1$ & 0.07 & 0.0656 & 0.0656 & 0.06629 & 0.0660 \\
$\mathrm{X} 2$ & 0.2 & 0.1691 & 0.1691 & 0.16913 & 0.1692 \\
$\mathrm{X} 3$ & 0.7 & 0.4539 & 0.5349 & 0.45394 & 0.4545 \\
$\mathrm{X} 4$ & 2.0 & 0.9113 & 0.9113 & 0.91132 & 0.9199 \\
$\mathrm{X} 5$ & 7.0 & 1.8954 & 1.8905 & 1.89545 & 1.9 \\
$\mathrm{X} 6$ & 20.0 & 3.3539 & 3.3539 & 3.35389 & 3.3563 \\
$\mathrm{X} 7$ & 70.0 & 6.4622 & 6.4622 & 6.46221 & 6.4444 \\
\hline
\end{tabular}

nanofluid towards a vertical stretching surface. It is observed that the skin friction coefficient reduces due to the increase in either of the nanoparticle buoyancy ratio or power-law fluid index whereas the effect reverses for the Brownian motion parameter, thermophoresis parameter, mixed convection parameter and velocity ratio. Furthermore, it is found that the increase in the Brownian motion parameter have enhancing effects on the local Nusselt number, while the increase in the thermophoresis parameter falls significantly the Nusselt number. Also, increases in the values of either of the velocity ratio or the power-law fluid index produced enhances in the local Nusselt number whereas, the opposite behavior was predicted as the mixed convection parameter and nanoparticle buoyancy ratio were increased for which the local Nusselt number was depressed. These results demonstrate the novelty of the current work which can be summarized as hybridization of group method analysis and rough set theory to use in the field of fluid dynamics effectively.
The proposed technique has simplified logic-based rules required to effectively predict these values with high accuracy and may be valuable in many engineering applications and be considered as knowledge base.

\section{Conclusion}

An extension work of using rough sets with other intelligent systems like neural networks, genetic algorithms, fuzzy approaches, and so forth, will be considered in the future work.

\section{Conflicts of Interest}

The author declare no conflict of interest

\section{Acknowledgments}

The author thank Prince Sattam bin Abdulaziz University, Deanship of Scientific Research at Prince Sattam bin Abdulaziz University for their continuous support and encouragement. 


\section{References}

[1] G. Astrita and G. Marrucci, "Principles of NonNewtonian Fluid Mechanics", McGraw-Hill, London, 1974.

[2] V. Shenoy and R. A. Mashelkar, "Thermal Convection in Non-Newtonian Fluids", $A d v$. Heat Transf., Vol. 15, pp. 143-225, 1982.

[3] J. T. F. Irvine and J. Karni, "Non-Newtonian fluid flow and heat transfer". In: Handbook of Single-Phase Convective Heat Transfer, New York: Wiley, 1987.

[4] M. J. Haung and B. L. Lin, "Mixed Convection from a Vertical Plate to Power Law Fluids", $J$. Thermophys. Heat Transf., Vol. 7, pp. 171-173, 1993.

[5] H. A. Nabwey, M. Boumazgour, and A. M. Rashad, "Group method analysis of mixed convection stagnation-point flow of nonNewtonian nanofluid over a vertical stretching surface", Indian Journal of Physics, Vol. 91, No. 7, pp. 731-742, 2017.

[6] S. M. M. EL-Kabeir, A. J. Chamkha, and A. M. Rashad, "Heat and Mass Transfer by MHD Stagnation-Point Flow of a Power-Law Fluid towards a Stretching Surface with Radiation, Chemical Reaction and Soret and Dufour Effects", International Journal Of Chemical Reactor Engineering, Vol. 8, A132. 2010.

[7] S. Choi and J. Eastman, "Enhancing thermal conductivity of fluids with nanoparticles", In: No. ANL/MSD/CP-84938; CONF-951135-29, Argonne National Lab, United States, 1995.

[8] S. A. Angayarkanni and J. Philip, "Review on thermal properties of nanofluids: Recent developments", Advances in Colloid and Interface Science, Vol. 225, pp. 146-176, 2015.

[9] J. Buongiorno, D. C. Venerus, N. Prabhat, T. McKrell, J. Townsend, R. Christianson, Y. Tolmachev, P. Keblinski, W. Hu, J. L. Alvarado, and I. C. Bang, "A benchmark study on the thermal conductivity of nanofluids", Journal of Applied Physics, Vol. 106, No. 9, p. 094312, 2009

[10] J. Buongiorno, "Convective transport in nanofluids", ASME J Heat transf, Vol. 128, pp. 240-50, 2006.

[11] I. Tlili, H. A. Nabwey, S. P. Samrat, N. sandeep, "3D MHD nonlinear radiative flow of $\mathrm{CuO}$ $\mathrm{MgO} /$ methanol hybrid nanofluid beyond an irregular dimension surface with slip effect", $S c i$ Rep, Vol. 10, 9181, 2020.

[12] Tlili, H. A. Nabwey, G. P. Ashwinkumar, and N. sandeep, "3-D magnetohydrodynamic AA7072AA7075/methanol hybrid nanofluid flow above an uneven thickness surface with slip effect", $S c i$ Rep, Vol. 10, p. 4265, 2020.

[13] R. K. Tiwari and M. K. Das, "Heat transfer augmentation in a two-sided lid-driven differentially heated square cavity utilizing nanofluids", Int. J Heat Mass Tran, Vol. 50, pp. 2002-2018., 2007.

[14] M. Ferdows, Z. Khairy, A. M. Rashad, and H. A. Nabwey, "MHD Bioconvection Flow and Heat Transfer of Nanofluid through an Exponentially Stretchable Sheet", Symmetry, Vol. 12, No. 5, 692,2020

[15] A. Zahra, S. U. Khan, H. Waqas, H. A. Nabwey, and I. Tlili, "Utilization of second order slip, activation energy and viscous dissipation consequences in thermally developed flow of third grade nanofluid with gyrotactic microorganisms", Symmetry, Vol. 12, No. 2, 309, 2020.

[16] H. A. Nabwey, "A revised model of Steady laminar natural convection of nanofluid under the impact of magnetic field on two-dimensional cavity with radiation [AIP advances 9, 065008 (2019); https://doi. Org/10.1063/1.5109192].", Thermal Science, Vol. 24, No. 1, pp. $421-425,2020$.

[17] A. Mahdy, A. J. Chamkha, and H. A. Nabwey, "Entropy analysis and unsteady MHD mixed convection stagnation-point flow of Casson nanofluid around a rotating sphere", Alexandria Engineering Journal, Vol. 59, No. 3, pp. 16931703, 2020.

[18] R. Gorla, S. M. M. El-Kabeir, and A. M. Rashad, "Heat transfer in the boundary layer on a stretching circular cylinder in a nanofluid", Journal of Thermophysics and Heat Transfer, Vol. 25, No. 1, pp. 183-186, 2011.

[19] A. J. Chamkha, M. Modather, S. M. M ELKabeir, and A. M. Rashad, "Radiative effects on boundary-layer flow of a nanofluid on a continuously moving or fixed permeable surface", Recent Patents on Mechanical Engineering, Vol. 5, No. 3, pp. 176-183, 2012.

[20] Tlili, N. Sandeep, M. G. Reddy, and H. A. Nabwey, "Effect of radiation on engine oil$\mathrm{TC} 4 / \mathrm{NiCr}$ mixture nanofluid flow over a revolving cone in mutable permeable medium", Ain Shams Engineering Journal, 2020.

[21] N. Khan, H. A. Nabwey, M. S. Hashmi, S. U. Khan, and I. Tlili, "A theoretical analysis for mixed convection flow of Maxwell fluid between two infinite isothermal stretching disks with heat source/sink", Symmetry, Vol. 12, No. 1, 62, 2020. 
[22] N. Imran, M. Javed, M. Sohail, T. Phatiphat, H. A. Nabwey, and I. Tlili, "Utilization of hall current and ions slip effects for the dynamic simulation of peristalsis in a compliant channel", Alexandria Engineering Journal, 2020.

[23] A. M. Rashad and H. A. Nabwey, "Gyrotactic mixed bioconvection flow of a nanofluid past a circular cylinder with convective boundary condition", Journal of the Taiwan Institute of Chemical Engineers, Vol. 99, pp. 9-17, 2019.

[24] A. J. Chamkha, H. A. Nabwey, Z. M. A. Abdelrahman, and A. M. Rashad, "Mixed bioconvective flow over a wedge in porous media drenched with a nanofluid", Journal of Nanofluids, Vol. 8, No. 8 , pp. 1692-1703, 2019.

[25] H. A. Nabwey, "Feasibility of Rough Sets Theory in Predicting Heat Transfer Performance in Thermally Developed Flow of Third Grade Nanofluid with Gyrotactic Microorganisms", Journal of Nanofluids, Vol. 9, No.1, pp.66-74, 2020.

[26] A. JJ. Chamkha, A. M. Rashad, A. I. Alsabery, Z. M. A. Abdelrahman, and H. A. Nabwey, "Impact of partial slip on magneto-ferrofluids mixed convection flow in enclosure", Journal of Thermal Science and Engineering Applications, Vol. 12, No. 5, pp. 1-13, 2020.

[27] A. JJ. Chamkha, R. Yassen, M. A. Ismael, A. M. Rashad, T. Salah, and H. A. Nabwey, "MHD Free Convection of Localized Heat Source/Sink in Hybrid Nanofluid-Filled Square Cavity", Journal of Nanofluids, Vol. 9, No. 1, pp. 1-12, 2020.

[28] Mahdy, F. M. Hady, and H. A. Nabwey, "Unsteady homogeneous-heterogeneous reactions in MHD nanofluid mixed convection flow past a stagnation point of an impulsively rotating sphere", Thermal Science, 388-388, 2019

[29] S. M. M. El-Kabeir, A. J. Chamkha, and A. M. Rashad, "Effect of thermal radiation on nonDarcy natural convection from a vertical cylinder embedded in a nanofluid porous media", Journal of Porous Media, Vol. 17, No. 3, pp. 269-278, 2014

[30] A. Y. Bakier, A. M. Rashad, and M. A. Mansour, "Group method analysis of melting effect on MHD mixed convection flow from radiate vertical plate embedded in a saturated porous media", Communications in Nonlinear Science and Numerical Simulation, Vol. 14, No. 5, pp. 2160-2170, 2009.

[31] H. A. Nabwey, W. A. Khan, and A. M. Rashad, "Lie Group Analysis of Unsteady Flow of
Kerosene/Cobalt Ferrofluid Past A Radiated Stretching Surface with Navier Slip and Convective Heating", Mathematics, Vol. 8, No. 5, 826, 2020.

[32] M. Ferdows, H. A. Nabwey, A. M. Rashad, M. J. Uddin, and F. Alzahrani, "Boundary layer flow of a nanofluid past a horizontal flat plate in a Darcy porous medium: A Lie group approach", In: Proc. of the Institution of Mechanical Engineers, Part C: Journal of Mechanical Engineering Science, Vol. 234, No. 8, pp.1545-1553, 2020.

[33] H. A. Nabwey, and H. A. El-Mky, "Lie group analysis of thermophoresis on a vertical surface in a porous medium", Journal of King Saud University-Science, Vol. 31, No. 4, pp. 10481055, 2019.

[34] S. M. M. El-Kabeir, M. A. El-Hakiem, and A. M. Rashad, "Group method analysis for the effect of radiation on MHD coupled heat and mass transfer natural convection flow water vapor over a vertical cone through porous medium", International Journal of Applied Mathematics and Mechanics, Vol. 3, No. 2, pp. 35-53, 2007.

[35] H. A. Nabwey, S. M. M. El-Kabeir, and A. M. Rashad, "Lie group analysis of effects of radiation and chemical reaction on heat and mass transfer by unsteady slip flow from a nonisothermal stretching sheet immersed in a porous medium", Journal of Computational and Theoretical Nanoscience, Vol. 12, No. 11, pp. 4056-4062, 2015

[36] M. M. Rashidi, E. Momoniat, M. Ferdows, and A. Basiriparsa, "Lie group solution for free convective flow of a nanofluid past a chemically reacting horizontal plate in a porous media", Mathematical Problems in Engineering, Vol. 2014, 239082, 2014.

[37] Z. Pawlak, "On learning — a rough set approach", In Symposium on Computation Theory, Springer, Berlin, Heidelberg, pp. 197-227, 1984.

[38] H. A. Nabwey, "A Hybrid Approach for Extracting Classification Rules Based on Rough Set Methodology and Fuzzy Inference System and Its Application in Groundwater Quality Assessment”, In: Advances in Fuzzy Logic and Technology, Springer, Cham, pp. 611-625, 2017.

[39] H. A. Nabwey, M. Modather, and M. Abdou, "Rough set theory based method for building knowledge for the rate of heat transfer on free convection over a vertical flat plate embedded in a porous medium", In: Proc. of International Conf. on Computing, Communication and Security (ICCCS), IEEE, pp. 1-8, 2015. 
[40] H. A. Nabwey, "An approach based on Rough Sets Theory and Grey System for Implementation of Rule-Based Control for Sustainability of Rotary Clinker Kiln", International Journal of Engineering Research and Technology, Vol. 12, No. 12, pp. 2604-2610, 2019.

[41] S. M. Shaaban and H. A. Nabwey, "A decision tree approach for steam turbine-generator fault diagnosis", International Journal of Advanced Science and Technology, Vol. 51, pp. 59-66, 2013.

[42] S. M. Shaaban and H. A. Nabwey, "A probabilistic rough set approach for water reservoirs site location decision making", In: Proc. of International Conf. on Computational Science and Its Applications, Springer, Berlin, Heidelberg, pp. 358-372, 2012.

[43] S. M. Shaaban, and H. A. Nabwey, "Rehabilitation and reconstruction of asphalts pavement decision making based on rough set theory." In: International Conference on Computational Science and Its Applications, Springer, Berlin, Heidelberg, pp. 316-330, 2012.

[44] S. M. Shaaban and H. A. Nabwey, "Transformer fault diagnosis method based on rough set and generalized distribution table", International Journal of Intelligent Engineering and Systems, Vol. 5, No. 2, pp. 17-24, 2012.

[45] H. A. Nabwey and M. S. El-Paoumy, "An integrated methodology of rough set theory and grey system for extracting decision rules", International Journal of Hybrid Information Technology, Vol. 6, No. 1, pp. 5765, 2013

[46] H. A. Nabwey, "An intelligent mining model for medical diagnosis of heart disease based on rough set data analysis", International Journal of Engineering Research and Technology, Vol. 13, No. 2, pp. 355-363. 2020.

[47] H. A. Nabwey, "Rough set approach for analyzing the effect of viscoelastic and micropolar parameters on hiemenz flow in hydromagnetics", International Journal of Engineering Research and Technology, Vol. 13, No. 1, pp. 170-180, 2020.

[48] H. A. Mohamed, "A probabilistic rough set approach to rule discovery", In: Proc. of International Conf. on Ubiquitous Computing and Multimedia Applications, Springer, Berlin, Heidelberg, pp. 55-65, 2011.

[49] H.A. Nabwey, "A Mathematical Methodology for Predicting the Primary Site of Metastatic adenocarcinoma Cancer based on Rough Set Theory", International Journal of Engineering
Research and Technology, Vol. 13, No. 3, pp. 427-432, 2020.

[50] H. K. Pathak, R. George, H. A. Nabwey, M. S. El-Paoumy, and K. P. Reshma, "Some generalized fixed point results in ab-metric space and application to matrix equations", Fixed Point Theory and Applications, Vol. 1, pp. 1-17, 2015.

[51] Nabwey, H. A., "A Methodology Based on Rough Set Theory and Hypergraph for the Prediction of Wart Treatment", International Journal of Engineering Research and Technology, Vol. 13, No.3, pp. 552-559, 2020.

[52] H. A. Nabwey, "Identification of short circuit fault location in voltage source inverters based on rough set theory", International Journal of Engineering Research and Technology, Vol. 13, No. 5, pp. 929-937, 2020.

[53] H. A. Mohamed, "An Algorithm for Mining Decision Rules Based on Decision Network and Rough Set Theory", In: Proc. of International Conf. on Ubiquitous Computing and Multimedia Applications, Springer, Berlin, Heidelberg, pp. 44-54, 2011.

[54] H. A. Nabwey, "A method for fault prediction of air brake system in vehicles", International Journal of Engineering Research and Technology, Vol. 13, No. 5, pp. 1002-1008, 2020.

[55] A. B. Metzner, "Heat transfer in non-Newtonian fluids", Advances in Heat Transfer, Vol. 2, pp. 357-397, 1965.

[56] M. J. Moran and R. A. Gaggioli, "Reduction of the number of variables in systems of partial differential equations, with auxiliary conditions", SIAM Journal on Applied Mathematics, Vol. 16, No. 1, pp. 202-215, 1968.

[57] Y. Wang, "Free convection on a vertical stretching surface", ZAMM Journal of Applied Mathematics and Mechanics/Zeitschrift für Angewandte Mathematik und Mechanik, Vol. 69, No. 11, pp. 418-420, 1989.

[58] R. S. R. Gorla and I. Sidawi, "Free convection on a vertical stretching surface with suction and blowing", Applied Scientific Research, Vol. 52, No. 3, pp. 247-257, 1994. 


\section{Nomenclature}

\begin{tabular}{|c|c|}
\hline$x$ and $y$ & The Cartesian Coordinates \\
\hline$U_{w}(x)=a x$ & the stretching velocity \\
\hline$U(x)=c x$ & the velocity of external flow \\
\hline$T_{\infty}$ & $\begin{array}{r}\text { the ambient temperature far away from the } \\
\text { surface }\end{array}$ \\
\hline$C_{\infty}$ & $\begin{array}{r}\text { The nanoparticle volume fraction far away } \\
\text { from the surface }\end{array}$ \\
\hline$C$ & nanoparticle volume fraction \\
\hline$(u, v)$ & the velocity components along the $(x, y)$ \\
\hline$T$ & the fluid temperature \\
\hline$\beta$ & $\begin{array}{r}\text { the volumetric expansion coefficient of the } \\
\text { fluid }\end{array}$ \\
\hline$g$ & gravitational acceleration \\
\hline$v$ & kinematic viscosity \\
\hline$k$ & thermal conductivity \\
\hline$\rho_{f}$ & fluid density \\
\hline$\rho_{p}$ & nanoparticles mass density \\
\hline$\alpha$ & thermal diffusivity of the nanofluid \\
\hline$C_{f}$ & the local skin friction \\
\hline$N u_{x}$ & local Nusselt number \\
\hline$\theta$ & Dimensionless temperature \\
\hline$\phi$ & Dimensionless nanoparticle volume fraction \\
\hline$\psi$ & Stream function \\
\hline$(\rho c)_{f}$ & the heat capacity of the fluid \\
\hline$(\rho c)_{p}$ & the effective heat capacity of the nanoparticle \\
\hline$D_{B}$ & the Brownian diffusion coefficient \\
\hline$D_{T}$ & the thermophoretic diffusion coefficient \\
\hline$K$ & consistency coefficient \\
\hline$n$ & the power-law fluid. \\
\hline$\lambda$ & $\begin{array}{r}\text { the dimensionless mixed convection } \\
\text { parameter }\end{array}$ \\
\hline$G r_{x}$ & the local Grashof number \\
\hline $\operatorname{Re}_{x}$ & the local Reynolds number \\
\hline $\mathrm{Nb}$ & the Brownian motion parameter \\
\hline$N t$ & the thermophoresis parameter \\
\hline $\operatorname{Pr}$ & the Prandtl number \\
\hline Le & the Lewis number. \\
\hline$a / c$ & ratio of velocity parameter \\
\hline$\Lambda$ & the mixed convection parameter \\
\hline $\mathrm{Nr}$ & buoyancy ratio \\
\hline
\end{tabular}

